\title{
Die Hausarztpraxis gegen einen Container tauschen
}

Peter Spirig

Korrespondenz:

Cornelia Mathis M. A. Eidgenössisches Departement für Verteidigung, Bevölkerungsschutz und Sport VBS Führungsstab der Armee / SWISSINT

Kasernenstrasse 8

CH-6370 Stans-Oberdorf

cornelia.mathis[at]vtg.admin.ch
Welche Ärztin, welcher Arzt hat nicht schon mindestens einmal davon geträumt, sein Joch bestehend aus Sprechstundenterminen, Fort- und Weiterbildungsverpflichtungen, Standespolitikveranstaltungen und nicht zuletzt Familienengagement abzustreifen und etwas völlig anderes $\mathrm{zu}$ unternehmen? Eine Art selbstgewähltes «Sabbatical» einzulegen? Diesen Kollegen kann geholfen werden. Sie können sich für einen Monat oder länger als Chief Medical Officer für einen Friedenseinsatz der Schweizer Armee engagieren.

Wir alle hängen an unserem Beruf und lieben ihn. Darum möchten wir nicht aussteigen. Dennoch ist ein «Tapetenwechsel» interessant, der die Möglichkeit eröffnet, den Beruf in einem fremden Land, in einer anderen Kultur und unter anderen Menschen auszuüben. Wer neugierig ist und genügend Schwung hat, sich einer speziellen Herausforderung zu stellen, für den ist ein solcher Einsatz massgeschneidert.

\section{Anforderungen entsprechen den Erfahrungen eines Allgemeinarztes}

Nach meinem Staatsexamen wollte ich als Schiffsarzt in die weite Welt hinaus. Es kam anders, weil mir in der Schweiz eine Traumstelle angeboten

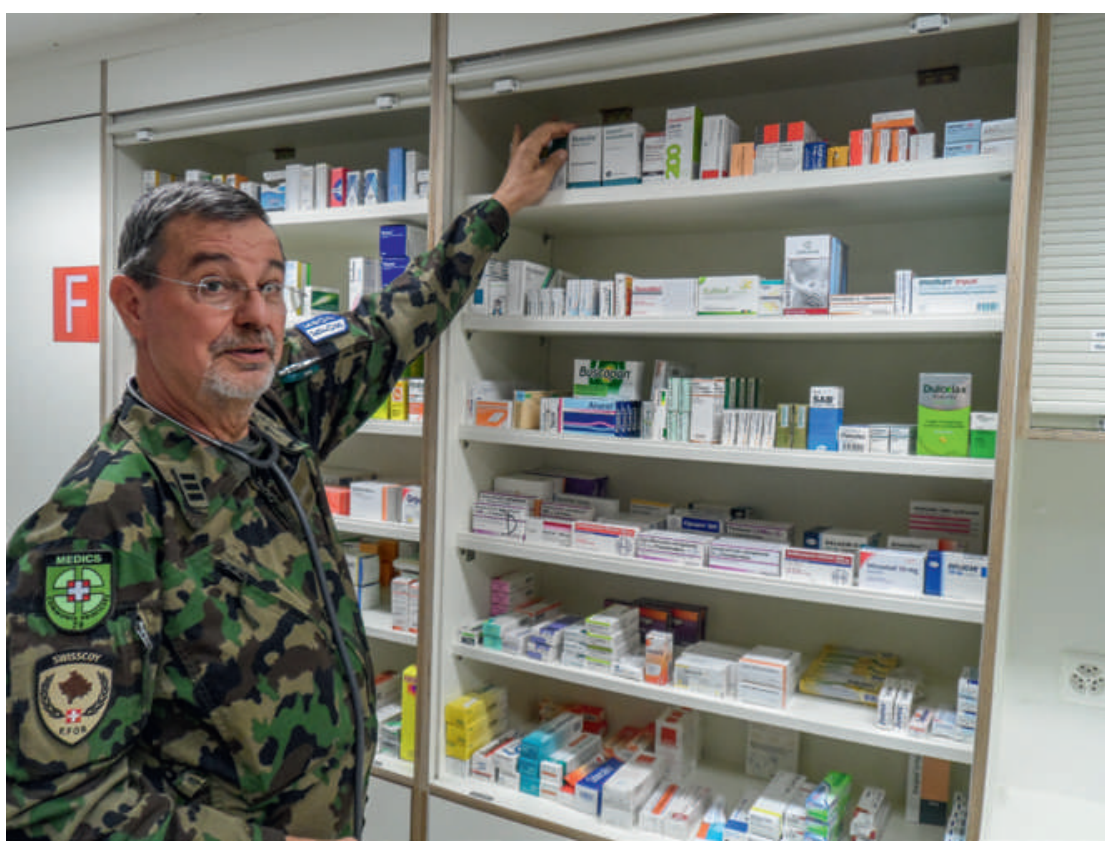

Dr. med Peter Spirig hat sich mit dem SWISSCOY-Einsatz als Militärarzt einen Lebenstraum erfüllt. wurde, auf die ich nicht verzichten konnte und wollte. Aber das Glück war mir hold, so dass ich am Ende meiner Karriere meinen Traum verwirklichen konnte. Ich war im Januar 2010 erstmals als Chief Medical Officer (CMO) im Kosovo für die SWISSCOY im Einsatz. Da ich diese Erfahrung als äusserst positiv erlebt hatte, sollten in den darauffolgenden Jahren weitere kürzere und längere Einsätze im Hauptquartier (HQ) der Kosovo Force (KFOR) in Pristina folgen.

Bevor es jedoch losging, wurden wir am Ausbildungszentrum des Kompetenzzentrums SWISSINT in Stans-Oberdorf für den Einsatz ausgebildet. Eine militärische Vorbildung ist nicht zwingend notwendig, stört aber keineswegs. Auch Ärztinnen ohne militärische Vorbildung können die einsatzbezogene Ausbildung beginnen. Während rund vier Wochen werden die nötigen Fähigkeiten, die für den friedensfördernden Auslandeinsatz zwingend sind, angeeignet.

Die beruflichen Anforderungen, die an einen Arzt im friedensfördernden Auslandeinsatz gestellt werden, entsprechen der Ausbildung und Erfahrungen eines Allgemeinarztes. Erfreulicherweise verletzten sich die Soldaten im Einsatz nur selten während der Ausführung ihrer Tätigkeiten. So stellt man in der täglichen Sprechstunde Diagnosen anderer Art: Weichteilverletzungen oder einfache Frakturen der Extremitäten aufgrund von Sportunfällen, Bienenstiche, Zahnschmerzen und saisonal bedingt internistische Fälle wie Erkältungen, grippale Infekte bis hin zur Pneumonie.

\section{Ein internationales Ärzteteam stärkt den Rücken}

Bei schweren Unfällen oder zur Abklärung diffuser Differenzialdiagnosen ist der Militärarzt nicht vollständig auf sich allein gestellt. Er kann sich in der Sicherheit wiegen, ein starkes Team von internationalen Ärzten im Rücken zu haben. In Prizren, eine einstündige Autofahrt südlich von Pristina entfernt, befindet sich das Einsatzlazarett der Deutschen Bundeswehr. Es ist vergleichbar mit einer Universitätsklinik und bietet genug Platz für stationäre Patienten. In diesem Feldlager sind auch Angehörige der Schweizer Truppen und Österreicher stationiert. So unterstützen sich die trinationalen Ärzte- und Pflegeteams gegenseitig und übernehmen gerne die Weiterbehandlung, wenn diese notwendig ist. Nur im äussersten Notfall muss ein Patient repatriiert, also in die Heimat geflogen werden. Zurück in das HQ der KFOR: In der Allgemeinpraxis, dem Medical 


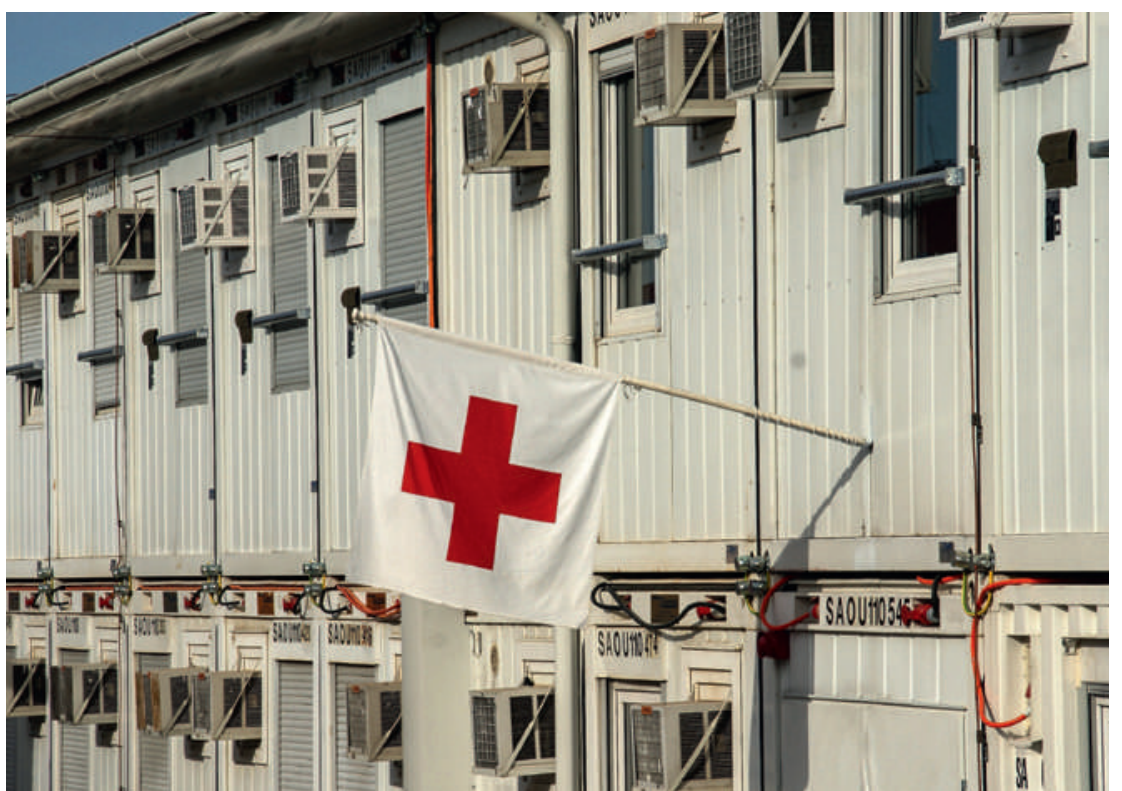

Das Schweizer-österreichische Medical Center im Hauptquartier der KFOR ist mit einer Hausarztpraxis zu vergleichen.

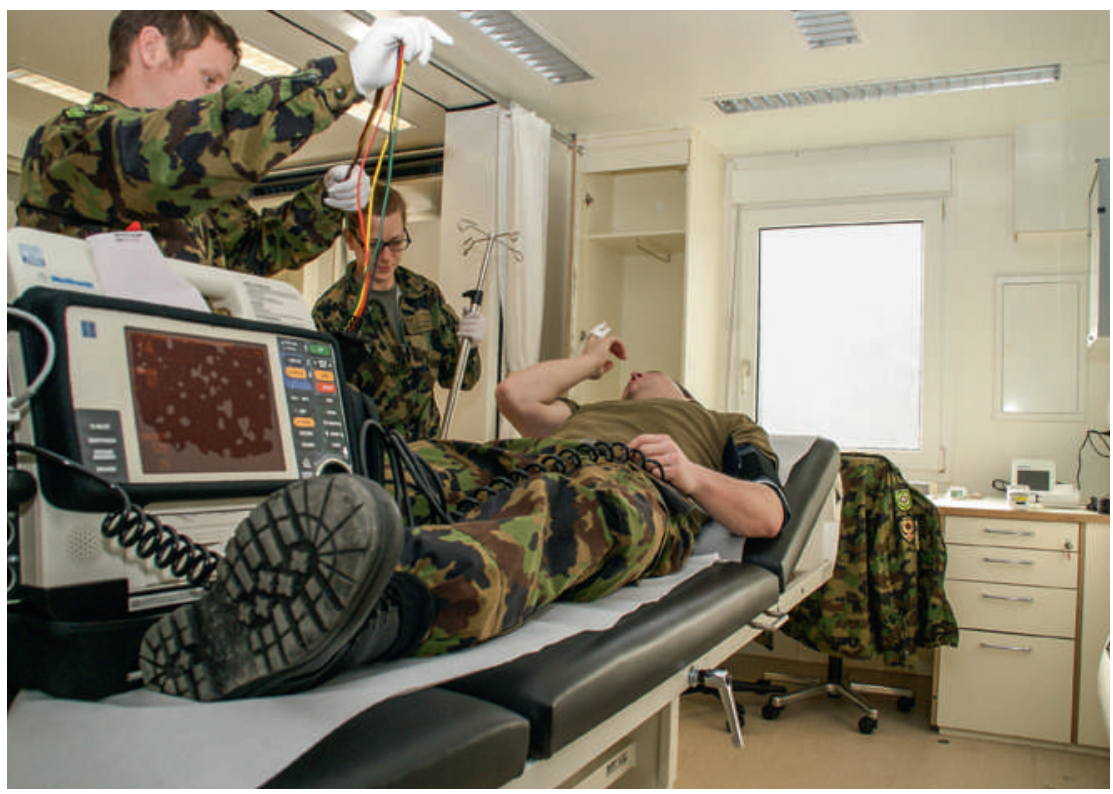

Die Kampfstiefel dürfen anbehalten werden - ein Soldat wird von medizinischem Personal überwacht. österreichischen Armeeangehörigen zu. Gelegentlich suchen auch deutsche Kameraden Hilfe im Medical Center, da sie die Möglichkeit haben, ihr Bedürfnis in ihrer Muttersprache zu formulieren. In einem medizinischen Notfall werden selbstverständlich auch Patienten anderer Nationen und Einheimische behandelt. Die KFOR setzt sich aus 31 Mitgliedsländern zusammen. Im Hauptquartier befindet sich neben dem Schweizer Medical Center eine weitere medizinische Einrichtung, die für alle Nationen zugänglich ist. Diese wird immer von der Nation betrieben, die den Kommandanten der KFOR stellt. Derzeit ist Italien die «Lead-Nation». Es finden auch Fort- und Weiterbildungen zwischen den Ärzten der einzelnen Nationen statt. Die Kontaktaufnahme zu anderen Ärzten hängt von der eigenen Initiative ab. Diese Zusammenarbeit ist zweifellos interessant, spannend und befriedigt die eingangs erwähnte Neugierde.

\section{Horizonterweiterung der besonderen Art}

Jeder wissensdurstige und unternehmungslustige Arzt, jede interessierte Ärztin, die sich von etwas Unkonventionellem herausgefordert fühlen, sammeln nicht nur medizinische Erfahrungen, sondern gewinnen auch auf menschlicher, sozialer und ethnischer Ebene. Von dieser Horizonterweiterung profitieren aber auch die zu Hause gelassenen Freunde und Familienangehörigen auf ungewöhnliche und unerwartete Art. Als persönlicher Ratschlag unter Kolleginnen und Kollegen empfehle ich wärmstens einen solchen Einsatz, den mit Sicherheit keine Ärztin und kein Arzt bereuen werden. Ansonsten stehe ich 24 Stunden für Reklamationen zur Verfügung. (Die Telefonnummer ist SWISSINT bekannt.)

Haben wir Ihr Interesse geweckt?

Das Kompetenzzentrum SWISSINT rekrutiert medizinisches Personal für einen sechsmonatigen Einsatz in der militärischen Friedensförderung. Auf www.armee.ch/peace-support erfahren Sie mehr. Wir freuen uns über Ihre Bewerbung: recruit.swisspso[at]vtg.admin.ch. Unter folgender Telefonnummer erhalten Sie genauere Auskunft: 0584675886

bestehend aus einem Arzt, einer Chief Nurse und ner weiteren Pflegefachperson auch noch österreichische Kolleginnen und Kollegen. Die Zusammenarbeit ist geradezu vorbildlich: Gleiche medizinische Standards wie auch die gemeinsame Sprache tragen der guten Zusammenarbeit bei.

\section{Ein Blick über den Tellerrand:}

\section{Fachgespräche mit Ärzten aus ganz Europa}

Die medizinische Versorgung des erwähnten Medical Centers steht in erster Linie den Schweizer und

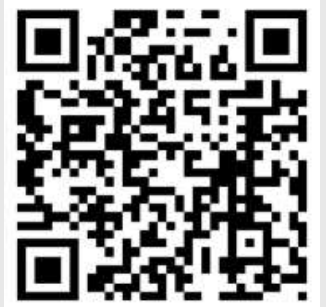

